\title{
A step-wise steerable source of illumination for low-noise 'Violin- Mode' shadow sensors, intended for use in interferometric gravitational wave detectors
}

\author{
N.A. Lockerbie, K.V. Tokmakov \\ SUPA (Scottish Universities Physics Alliance), Department of Physics, \\ University of Strathclyde, 107 Rottenrow, Glasgow G4 0NG, UK
}

\begin{abstract}
A steerable low-noise source of illumination is described for shadow-sensors having a displacement sensitivity of $\sim 100$ picometres $(\mathrm{rms}) / \sqrt{ } \mathrm{Hz}$, at $500 \mathrm{~Hz}$, over a measuring span of at least $\pm 0.5 \mathrm{~mm}$. These sensors were designed to detect lateral 'Violin-Mode' resonances in the highlytensioned fused-silica suspension fibres of the test-masses/mirrors for the Advanced LIGO (Laser Interferometer Gravitational wave Observatory) gravitational wave detectors. The shadow sensors-one intended for each of the four fibres in a suspension-comprised a source of Near InfraRed (NIR) radiation (emitter), and a differential shadow-displacement sensor (detector), these bracketing the fibre under test. The suspension fibres themselves were approximately $600 \mathrm{~mm}$ long by $0.4 \mathrm{~mm}$ in diameter, and, when illuminated from the side, they cast narrow, vertical, shadows onto their respective detectors - these being located at an effective distance of 50 fibre diameters behind the axes of the fibres themselves. The emitter described here was designed to compensate for a significant degree of mechanical drift or creep over time in the mean position of its suspension fibre. This was achieved by employing five adjacent columns of $8 \times$ miniature NIR LEDs $(\lambda=890 \mathrm{~nm})$, with one column being activated at a time. When used in conjunction with a 'reverse Galilean' telescope, the LED sources allowed the collimated beam from the emitter to be steered azimuthally in fine angular increments $\left(0.65^{\circ}\right)$, causing the fibre's shadow to move laterally, in a step-wise manner, across the plane of its facing detector. Each step in shadow position was approximately $0.23 \mathrm{~mm}$ in size, and this allowed the fibre's shadow to be re-centred, so as to bridge once again both elements of its photodiode detector-even if the fibre was off-centred by as much as $\pm 0.5 \mathrm{~mm}$. Re-centring allowed Violin-Mode vibrations of the fibre to be sensed once again as differential $A C$ photocurrents, these flowing in anti-phase in the two elements of the 'splitphotodiode' detector.
\end{abstract}

PACS numbers: 04.80.Nn, 84.30.-r, 06.30.Bp, 07.07.Df, 07.57.-c

\section{Introduction}

In the Advanced LIGO 'aLIGO' ground-based interferometric gravitational wave detectors, each $40 \mathrm{~kg}$ test-mass/mirror is suspended in vacuum from a multi-stage pendulum seismic isolation system [1-4]. Four fused-silica suspension fibres, each measuring approximately $600 \mathrm{~mm}$ long, by $0.4 \mathrm{~mm}$ in diameter, suspend the test-mass/mirror itself, thereby creating the lowest pendulum isolation stage for each test-mass $[4,5]$.

These thin suspension fibres are under very high strain, and the fundamental frequencies of their transverse 'Violin-Mode'(VM) vibrations are $\sim 500 \mathrm{~Hz}$, whilst the Q-values of these modes are exceptionally high $\left(\sim 6 \times 10^{8}[6]\right)$. Therefore, once excited by seismic activity, internal relaxations of mechanical stress, etc., these modes, and their harmonics, can transfer vibrational energy to the suspended test-masses within the gravitational-wave detection bandwidth. Moreover, ring-down decays could endure for periods of days, potentially, during which time gravitational wave observations could not be made. Consequently, it was considered necessary to monitor these transverse fibre vibrations, i.e., vibrations parallel to the interferometer's beam-axis [7], with a view then to cold-damp any VM resonances [8].

A measuring system was therefore devised which illuminated each fused-silica suspension fibre from the side, using a collimated Near InfraRed (NIR) light beam, so as to cast a shadow of the fibre onto a facing photodiode-based shadow-sensor. The source of the low-noise illuminating NIR beam was a column of eight sub-miniature OP224 LEDs, with a peak emission 


\section{LIGO-P1500195-v2}

wavelength of $\lambda=890 \mathrm{~nm}[9,10]$. Each shadow-sensor was in the form of a 'synthesized splitphotodiode' detector, this having essentially no 'dead-band' between its two sensing elements [11]. The intention here was to allow a vibrating suspension fibre to cast its shadow across the central, common, edge of the sensor's two detection elements, PDa and PDb, as shown in plan view in Figure 1-with the illumination coming from source 3 in the Figure. In this way, any lateral VM vibration of the fibre would cause instantaneously an incremental increase (say) in the photocurrent of PDa, and a concomitant decrease in the photocurrent of PDb, as the vibrating shadow slid slightly off element PDa and onto element PDb. Naturally, half a cycle later the sense of flow of these incremental currents would be reversed. Indeed, if elements $\mathrm{PDa}$ and PDb had the same vertical height (measured parallel to the long-axis of their respective fibre), and similar responsivities (measured in $\mathrm{A} \cdot \mathrm{W}^{-1}$ ), then these alternating photocurrents would always be closely equal in magnitude, and opposite in phase, depending as they do just on the 'depth' of the shadow falling over their common edge, i.e., upon its contrast [11]. These two AC currents then could be converted into voltages via transimpedance amplifiers, be differenced (so as to double the VM signal), and finally be post-amplified, and detected. Thus, such a shadow sensor could in principle form the basis of a viable VM monitoring system. The VM amplifier connected to the sensors of this work functioned in the manner just described, but it also possessed two subsidiary outputs, these producing voltage analogues of the mean, steady-state, $D C$ photocurrents flowing in detector elements PDa and PDb [12]. These two outputs were used for initial alignment of the fibre's shadow onto the centre of the detector, and for calibrating the $D C$ (and hence, $A C$ ) responsivity of the detection system to incremental fibre displacement $[12,13]$. However, for the intended application a target sensitivity to fibre vibration of 100 picometres/ $\mathrm{VHz}$ at $500 \mathrm{~Hz}$ was necessary, and, in the event, this proved quite a challenging target to meet, using such a simple, differential, shadowsensing system.

Details of just such a VM detection system have been published already - this detection system having met (indeed, exceeded) the above-mentioned target sensitivity [9, 11-14]. However, in this published system the allowable position of the illuminated fibre was restricted, relative to that of the dedicated $V M$ sensor, to a usable range of $\pm 0.1 \mathrm{~mm}$ about its central location. In contrast, the illumination source described here was designed to cater for a much larger range in mean position of the monitored fibre - a range of at least $\pm 0.5 \mathrm{~mm}$, in fact.

At the outset of this VM detector work it was felt that even if each shadow-sensor could be aligned correctly around its monitored fibre, initially, such that the fibre's shadow fell equally onto PDa and PDb, mechanical creep of the suspension's framework might lead nevertheless to a significant physical offset of the fibre from this central location. Indeed, it was feared that this offset might grow to be as large as $\pm 0.5 \mathrm{~mm}$, over time. However, a well-collimated incident (fibre-illuminating) beam is needed for good 'shadow depth,' since this maximizes the VM signal [11]; but then, of course, there is little spreading-out of the shadow cast by the fibre beyond the fibre's own geometrical diameter. Therefore, putting the potential offset figure of $\pm 0.5 \mathrm{~mm}$ into context: under a well-collimated incident beam, even a lateral creep in the fibre's mean position of just (a little over) $0.2 \mathrm{~mm}$ - the fibre's radius-would be enough to cause any VM signal to vanish, completely. This would come about because the fibre's shadow would no-longer straddle the common edge of its respective photodetector elements, PDa and PDb. Instead, the shadow would fall just over element PDa, or just over PDb. Consequently, lowlevel, lateral, VM vibration of the fibre and it attendant shadow could no-longer generate incrementally alternating photocurrents in either PDa, or PDb. Thus, for this type of VM detector, a fibre offset as large as $\pm 0.5 \mathrm{~mm}$ would be very serious, indeed.

\section{Step-wise repositioning of the fibre's shadow: the concept}

In the context of the stringent vacuum requirements of interferometric gravitational wave detectors, the use of moving actuators (piezo motors, for example) for the VM detectors would have been inappropriate. A simpler system having no moving parts therefore was devised, as 


\section{LIGO-P1500195-v2}

shown in Figure 1: an array of five, separate, well-collimated NIR beams was conceived, labelled 1-5 in the Figure, these being set at incrementally small angles to each other. By switching on

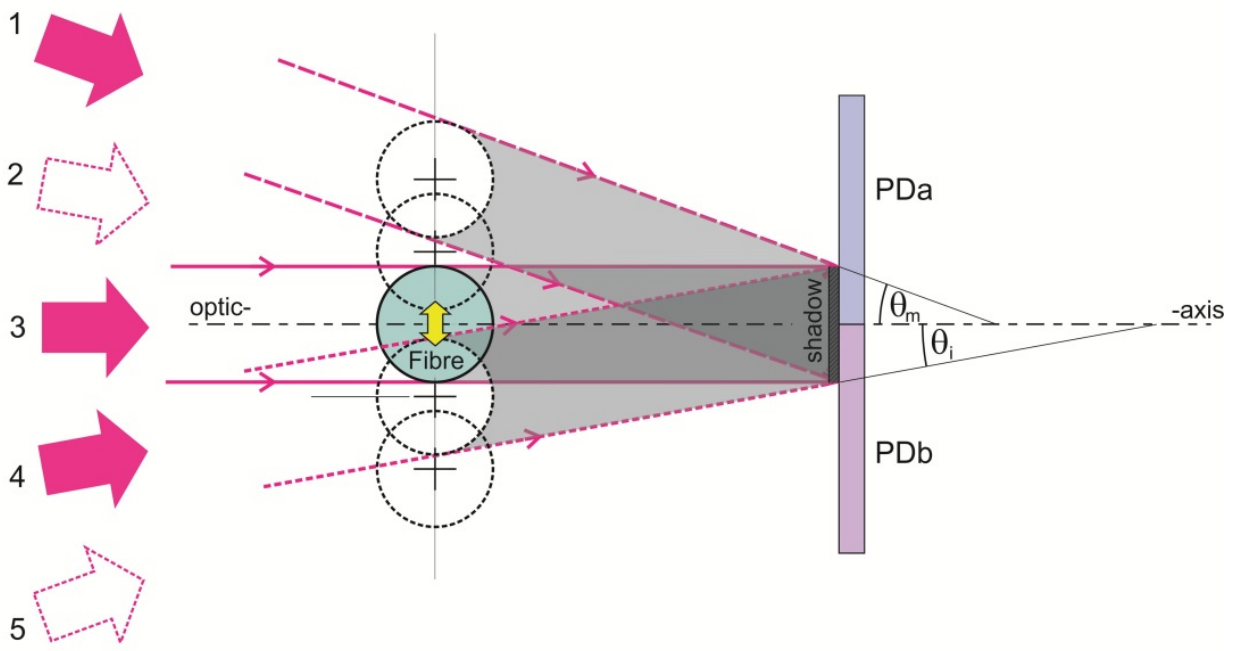

Figure 1. Concept for the step-wise repositioning of the shadow cast by an illuminated fused-silica suspension fibre. The Figure shows, in plan view, such a fibre being illuminated from the left by a collimated beam of Near InfraRed (NIR) radiation, this being directed along the indicated optic-axis (by source ' 3 '). This source of illumination casts a shadow of the fibre correctly onto its two shadow-displacement sensing elements, PDa and PDb. The double-headed arrow lying over the fibre's centre indicates $V M$ vibration of that fibre taking place parallel to the face of the detector. The four dashed circles illustrate creep over time in the fibre's mean position, in increments of $0.25 \mathrm{~mm}$, and the angled incident beams 2 and 1, and 4 and 5, show how the fibre's shadow nevertheless can be brought back so as to overlap the centre-line of the detector, as required: shadows from sources 1,3 , and 4 , have been drawn filled-in — to illustrate this action. For the geometry used, adjacent beams needed to be offset from each other by an angle of just $0.65^{\circ}$. Thus, the angle $\theta_{\mathrm{i}}=0.65^{\circ}$, and the angle of maximum correction $\theta_{\mathrm{m}}=1.3^{\circ}$. Clearly, these angles having been greatly exaggerated in the Figure.

\section{The optical arrangement for repositioning of the fibre's shadow}

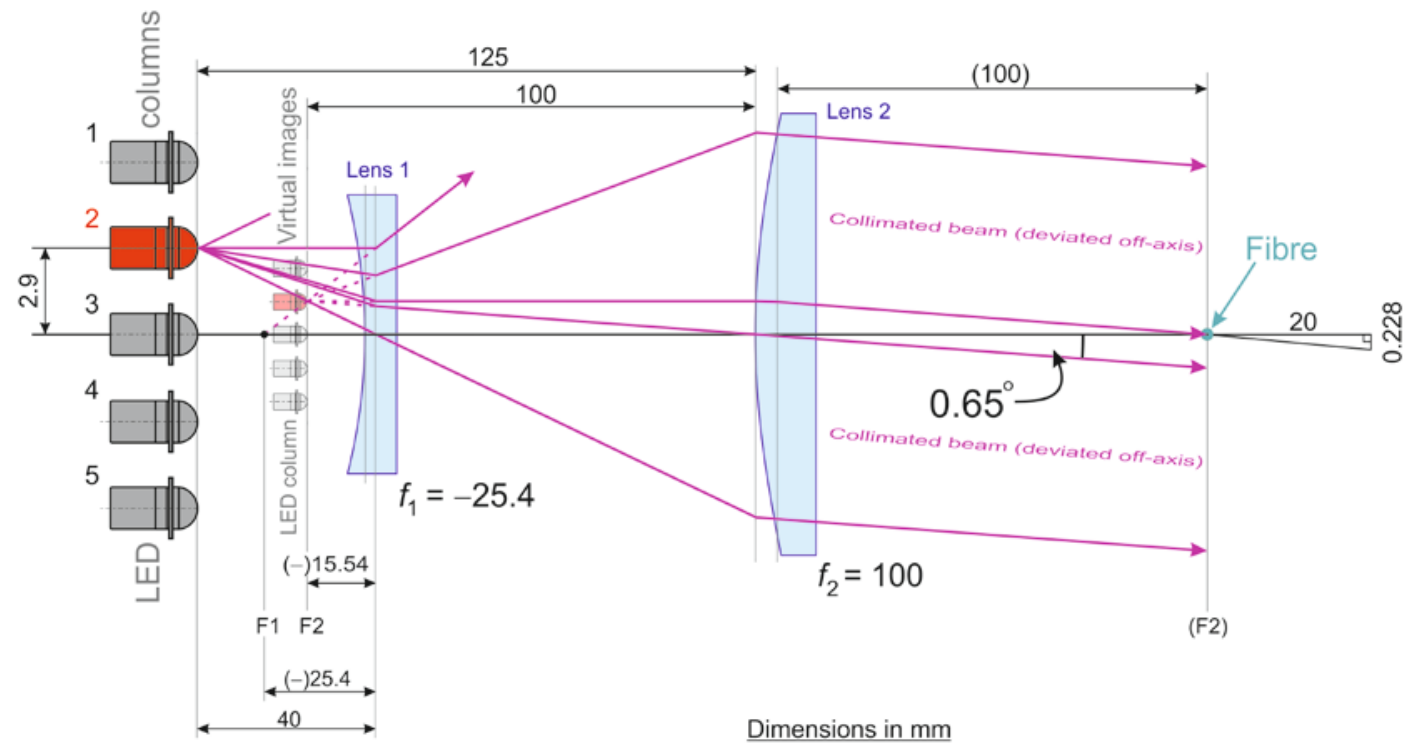

Figure 2. Schematic plan view of the 'reverse Galilean' telescopic arrangement for the step-wise rotation of the collimated NIR beam from the emitter. Five columns of LEDs, labelled 1-5 in the Figure, each comprised (effectively) $8 \times$ OP224 NIR LEDs. Column 2 is shown as being active. Lens 1 in the Figure was a planoconcave B270 crown glass cylindrical lens, with a focal length of (-) $25.4 \mathrm{~mm}$. This $40 \mathrm{~mm} \times 20 \mathrm{~mm}$ lens also served as a sealing window for the LEDs' housing. The diminished-in-width virtual images of the LED columns, viewed through Lens 1, are also indicated in the Figure. These served as the objects for collimating Lens 2, which was a planoconvex spherical lens, having a $100 \mathrm{~mm}$ focal length. This $40 \mathrm{~mm}$ diameter BK7 crown glass lens was cropped to a $25 \mathrm{~mm}$ width, so that two separate emitters could be used closely side-by-side, as in Figures 3 and 5. 


\section{LIGO-P1500195-v2}

these beams, one at a time, the fibre's shadow could be brought back so as to cover the centre of the 'split-photodiode' sensor, as indicated, even if the illuminated fibre had crept away from its central location (on the indicated optic-axis in the Figure) by as much as $\pm 0.5 \mathrm{~mm}$. In practice, however, because the fibre-to-sensor distance was equal to 50 fibre diameters (20 $\mathrm{mm}$ ), the necessary angles $\theta_{\mathrm{i}}$ and $\theta_{\mathrm{m}}$, shown schematically in the Figure, actually were rather small, at $0.65^{\circ}$ and $1.3^{\circ}$, respectively. Even using miniature lensed LEDs, such small angular deviations were not achievable, straightforwardly. This was due to the confined space available between the pairs of suspension fibres, which were themselves located on either side of each test-mass. A technique for achieving the required very small step-wise angular rotations of the emitter's output beam, within the confines of the available space, is shown schematically in plan-view, in Figure 2. It is effectively a Galilean telescope, used in reverse. Here, 5 closely-spaced columns of miniature, lensed, LEDs are made to appear physically even closer together by placing a diverging (cylindrical) lens in front of them-Lens 1 in the Figure. Lens 2, a longer focal length converging lens, collimates the light from each reduced-in-width virtual column of LEDs. Thus, by lighting each successive column of LEDs, one at a time, the emitter's collimated output beam can be rotated about a vertical line (i.e., in azimuth) in small angular steps-each of approximately $0.65^{\circ}$, for a $2.9 \mathrm{~mm}$ centre-to-centre spacing of the actual LED columns.

\section{The steerable 5-column 'reverse Galilean' Emitter}

A scale diagram of the apparatus, as constructed, is shown in Figure 3. Here, two emitters are shown illuminating a pair of suspension fibres for one side of a test-mass, these fibres being
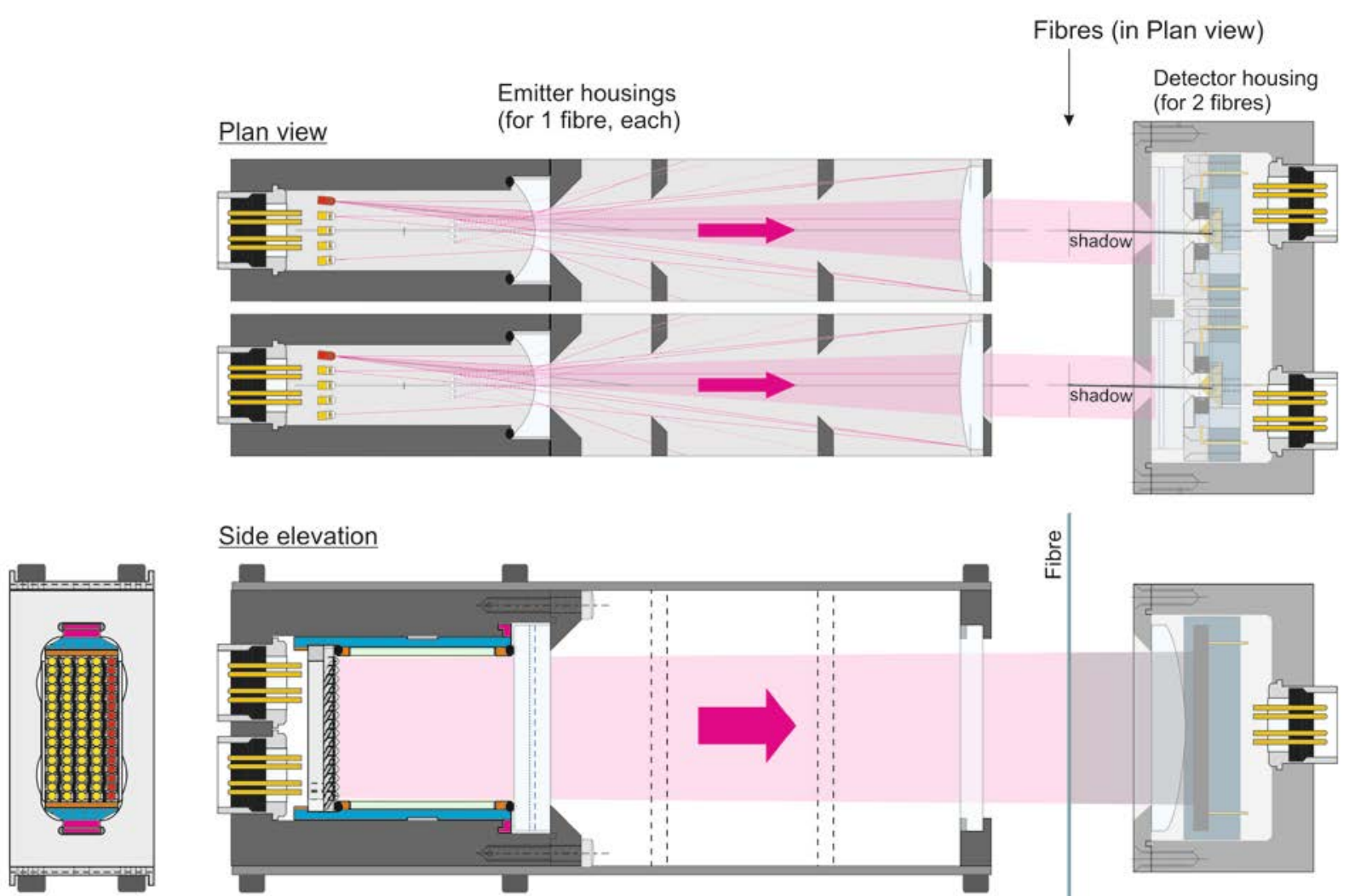

Side elevation

Front view of internal infrared LED array
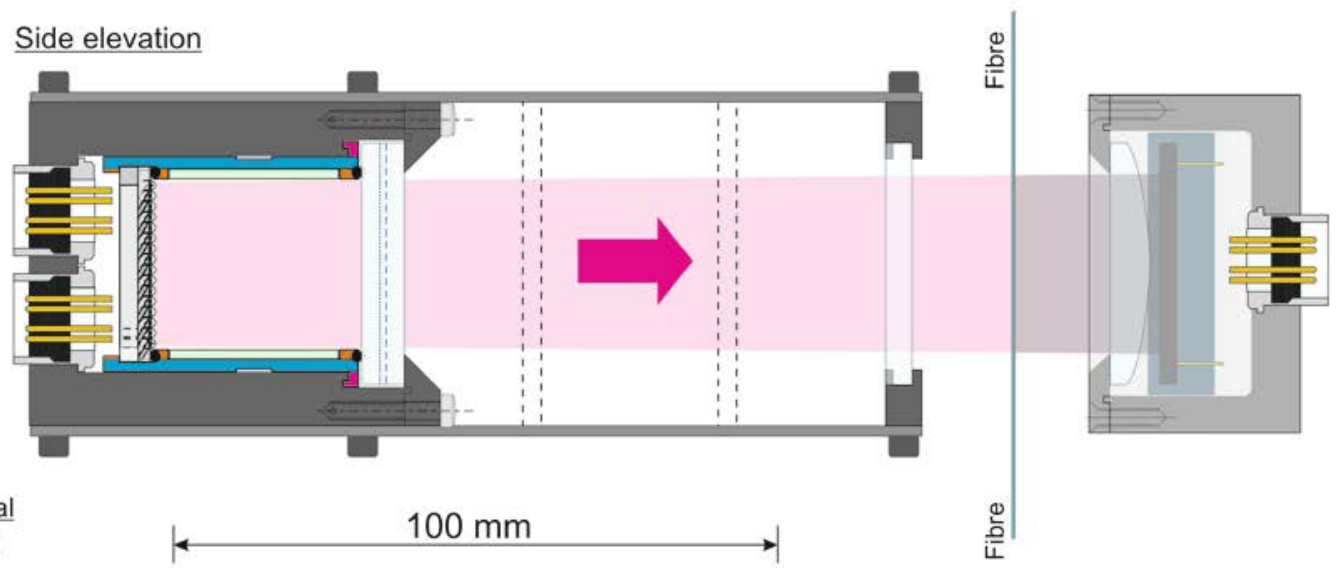

Figure 3. Scale diagram showing sectional views of a pair of emitters (only one was built and tested), here shown illuminating the two suspension fibres on one side of a suspended test-mass. The dual detector housing is shown on the right of the Figure: it contained two separate 'synthesized split-photodiode' detectors —one for each fibre. In each emitter housing, LED source (column) 1 is indicated as being fully lit, i.e., active. In fact, only 8 alternate LEDs in each column of 16 LEDs were switched on at a time (please refer to the text). 


\section{LIGO-P1500195-v2}

spaced a nominal $30 \mathrm{~mm}$ apart. In Figure 3 (plan, sectional, view), five adjacent columns of LEDs are shown within the housing of each emitter. In fact, only one such emitter was built, and the results from its operation are presented below.

Each column of miniature LEDs actually comprised $16 \times$ OP224 NIR devices, these being arranged in two strings of 8 series-connected LEDs (for redundancy-alternate LEDs belonged to the same string) [9]. The LEDs in each string were connected together electrically by preformed tin-plated copper strips, $0.1 \mathrm{~mm}$ thick. All 16 LEDs, and their connecting strips, were set into holders machined from insulating PEEK material, these holders measuring $2.6 \mathrm{~mm} \times$ $2.7 \mathrm{~mm} \times 32.3 \mathrm{~mm}$ tall. However, the overall height of each column of LEDs was $26 \mathrm{~mm}-$ measured over the LEDs, themselves. The photodiode sensor in the facing detector was reasonably well matched in size, being $29.1 \mathrm{~mm}$ tall [11]. The four outlying LED holders within each emitter housing were meant to be angled inwards towards their emitter's optic axis, as indicated in the plan view of Figure 3; but the addition of sheets of $70 \mu \mathrm{m}$ Kapton insulation, fitted outboard of columns 1 and 5 in order to insulate the contact strips from the emitter's close-fitting aluminium-alloy housing (as seen in the cut-away view of Figure 4), caused the beam-axis of these two outer sources to be aligned more nearly parallel with the optic-axis of the emitter, rather than being angled inwards. This offset resulted in a slight reduction in the detected intensity from these two columns, as discussed below.

Both strings of LEDs in a particular source (column) could be lit at the same time, but the results discussed below were obtained through lighting only one of the strings of 8 LEDs. However, in order to maximize the detected intensity from these LEDs-increasing it by approximately $40 \%$, in fact-two gold-on-chromium front-silvered glass mirrors were placed so as to bracket all 5 columns of 16 LEDs-from above and below-as shown in Figures 4 and 5. Their emission-enhancing effect can be thought of as being due to an increase in the apparent vertical height of the LED sources, via reflection from the mirrors at top and bottom.

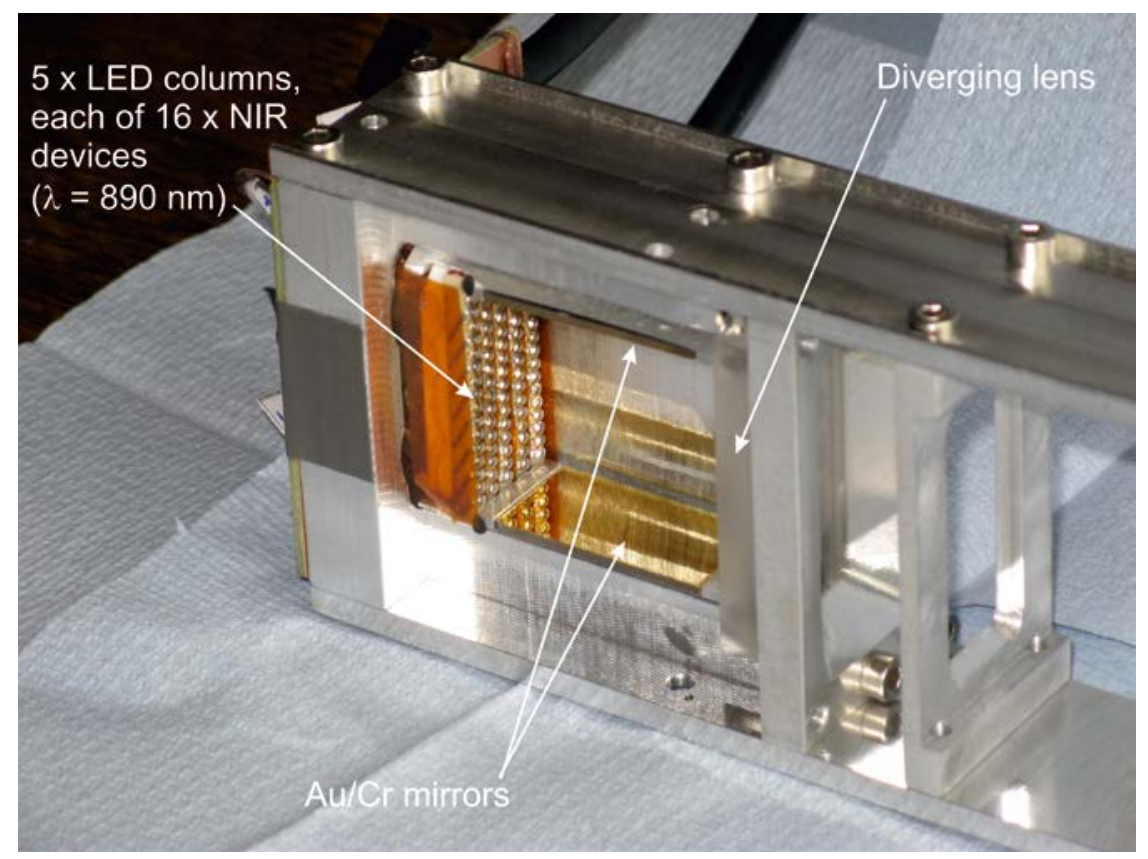

Figure 4. Cut-away view of the interior of the step-wise steerable 5-LED-column emitter. For the scale, please see Figure 6. There were $16 \times$ OP224 miniature, lensed, LEDs in each source (column), i.e., 80 LEDs in total. The front-silvered $\mathrm{Au} / \mathrm{Cr}$ mirrors, top and bottom, increased the emitted beam intensity (please refer to the text).

Figure 6 shows the as-built 5-column emitter, together with a fully-functioning dual-detector housing (as shown schematically in the drawings of Figure 3), and a steel rule, for scale. Figure 6 shows the emitter having been aligned with the nearer of the two synthesized splitphotodiode detectors contained within the detector housing. The Figure also shows the emitter 


\section{LIGO-P1500195-v2}

before one of its housing's walls was machined away to reveal its interior structure-seen in Figures 4 and 5.

The electronics for powering the 5 columns of LEDs in each of the four planned emitters, and for housing the four corresponding VM amplifiers, was built in-house, and it was mounted within a 19" rack-mounting enclosure, as seen in Figure 7. Here, the four suspension fibres of a Gravitational Wave Detector's test-masses/mirrors were designated A-D, and the Figure shows two $6 \mathrm{U}$ modules on the left and centre of the photo carrying four front-panel rotary-

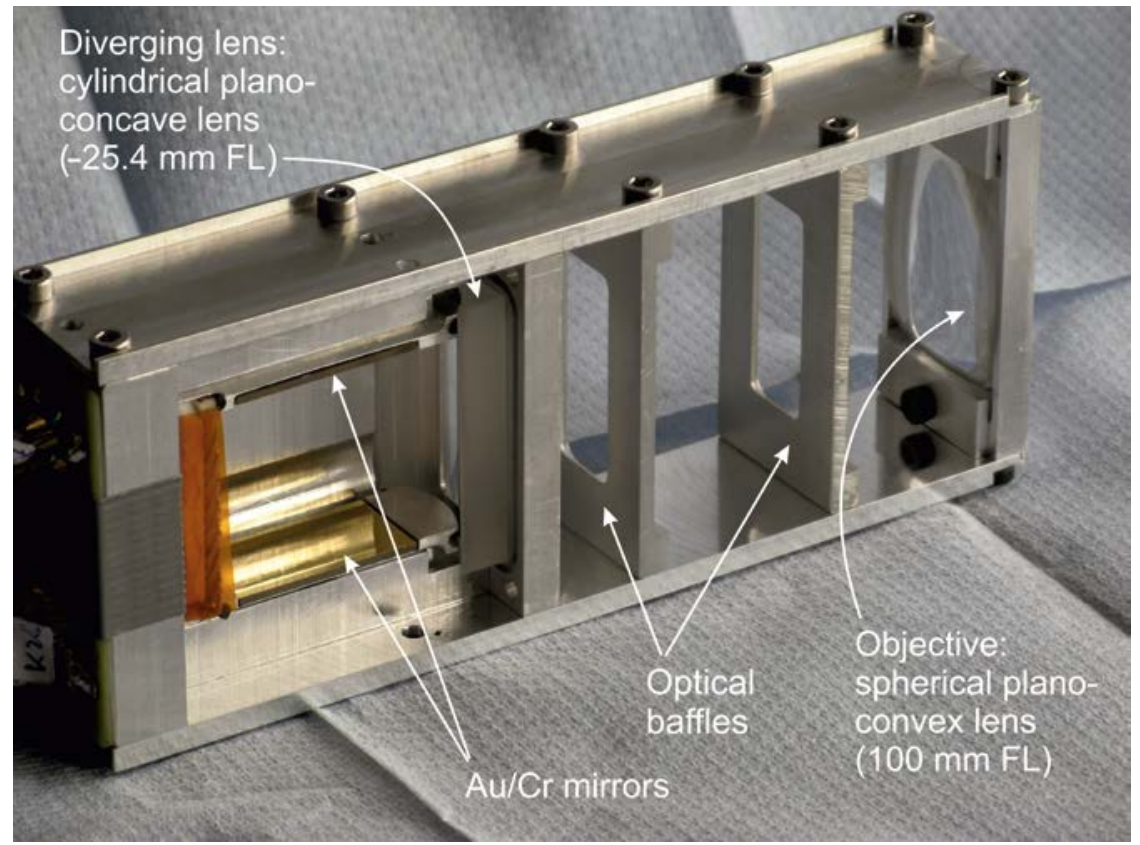

Figure 5. Cut-away view of the interior of the step-wise steerable 5-LED-column emitter, showing the planoconcave cylindrical lens (acting also as a vacuum-sealed window to the emitter housing), and the plano-convex spherical collimating lens. The optical baffles reduced lateral light leakage, and so interference between emitters and their adjacent detectors. For the scale, please see Figure 6.

switches, one dedicated to each prospective emitter. Each of these switches allowed a lownoise $25 \mathrm{~mA}$ current to be switched between the 5 sources (columns) of 8 LEDs in the

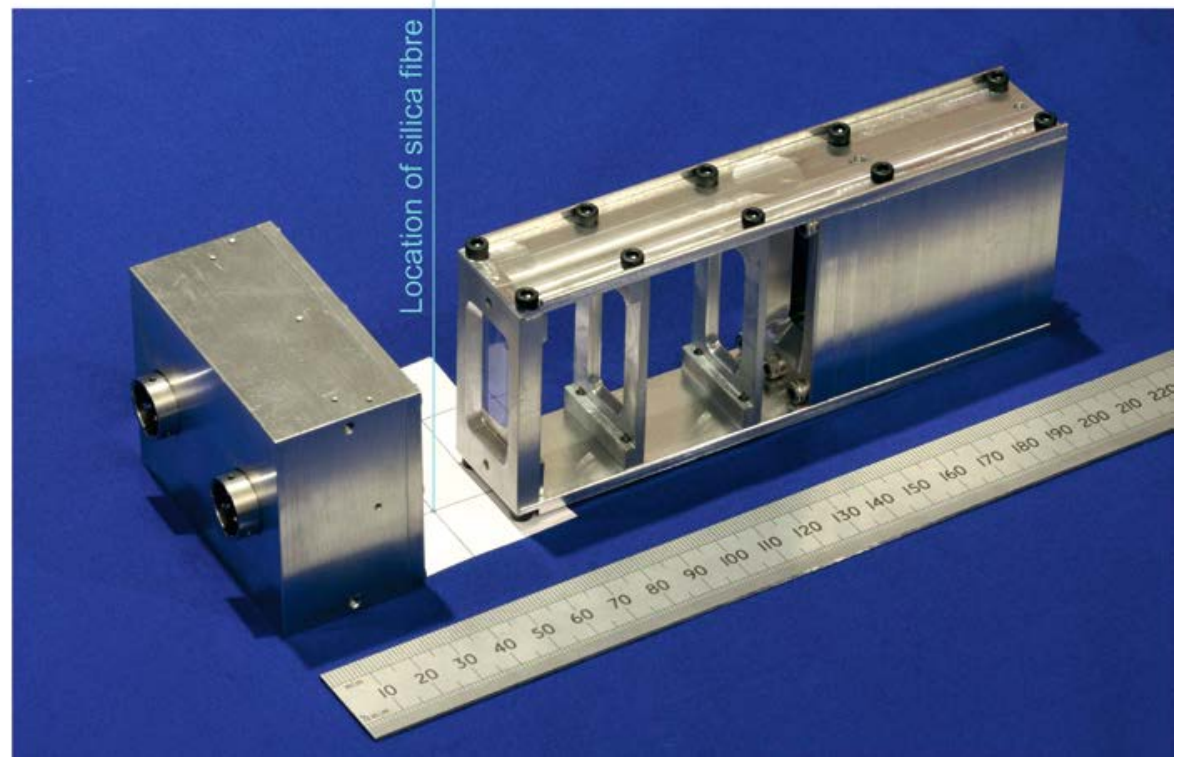

Figure 6. At left: dual-detector housing. Each of the two detectors in the housing was in the form of a 'synthesized split-photodiode.' At right: the as-built step-wise steerable 5-LED-column source of illumination, as shown in the sectional drawings of Figure 3, and in the cut-away photos of Figures 4 and 5 . The location of the (vertically orientated) $0.4 \mathrm{~mm}$ diameter fused-silica suspension fibre is indicated, this lying between the steerable source and the nearer of the two detectors. 


\section{LIGO-P1500195-v2}

respective emitter. The column-switching was set up to be controlled, and read-back, remotely-but the front-panel manual settings could be allowed to override this external control via a front-panel switch - a red witness LED on the panel showing which source (column) was currently active. The two 3U modules on the right of Figure 7 each contained two Violin-Mode detection amplifiers, one per suspension fibre (i.e., four amplifiers in total). These amplifiers had a transimpedance-gain of $120 \mathrm{k} \Omega$ at each of their $D C$ outputs (for PDa and, separately, for PDb), and a mid-band gain of $\sim 120 \mathrm{M} \Omega$ at their single $V M$ (differential) $A C$ output [12].

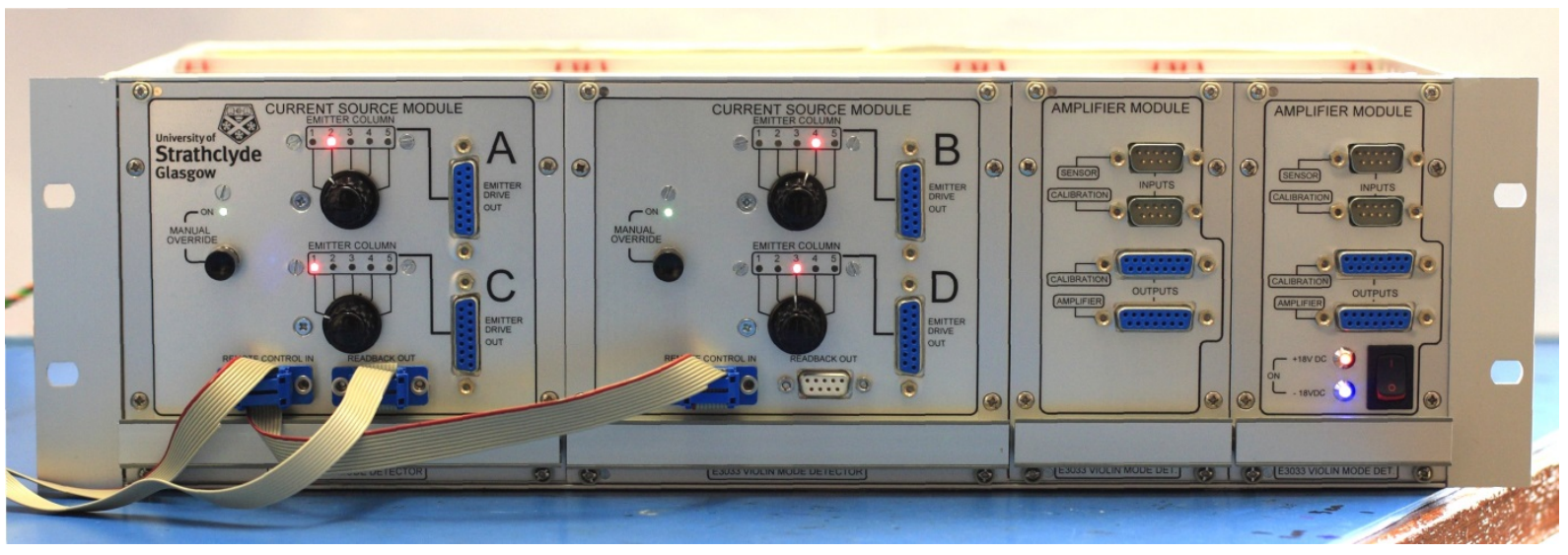

Figure 7. 19" rack-mounted electronics for controlling four step-wise steerable sources, and their corresponding Violin-Mode vibration detectors. Which of the 5 columns in each emitter (A-D) was lit was determined by the setting of the corresponding front-panel rotary switch-when the 'Manual Override' button was pressed. Otherwise, the column being lit was determined by an externally applied digital code. In both cases, which LED column was currently lit was mirrored by a red witness LED on the front panel (please refer to the text).

\section{Shadow-steering tests}

The 'column 1' LEDs of the 5-column emitter shown in Figure 6 were switched on, and a vertically-orientated silica suspension-fibre sample, $0.4 \mathrm{~mm}$ in diameter, was translated at a constant rate (of approximately $50 \mu \mathrm{m} \mathrm{s}^{-1}$ ) so as to pass along a direction similar to that indicated in Figure 3 by the arrow labelled 'Fibres (in Plan view)'. In this way, the fibre passed transversely through the emitter's NIR beam, such that its slowly moving shadow was cast onto the facing detector. This caused 'notches' to appear, in turn, in the two DC output signals, as the shadow passed firstly across photodiode element PDa, and then across PDb. The fibre sample was translated using a motorised sliding carriage, whose position was recorded via a magnetic encoder, this having a positional resolution of $\pm 1 \mu \mathrm{m}$. The encoder's magnetic track also incorporated a datum (zero) reference position [13]. The $D C$ outputs coming from the two photodiode detector elements were monitored as a function of fibre position, and then differentiated off-line. Next, these two derivative signals were differenced-so as to double the size of the measured $D C$ responsivity (rate of change of output voltage as a function of fibre position) [11,13].

This procedure then was repeated, with individual LED columns 2, 3, 4, and 5 being switched on, successively. In all cases, the current supply for the string of 8 LEDs in each column was a steady $25 \mathrm{~mA}$.

\section{Shadow-steering results}

The results of these tests are shown in Figures 8 and 9. From the original data, as shown by the solid traces in Figure 8, a DC responsivity of (at least) $3725 \mathrm{~V} / \mathrm{m}$ was achieved across the three central peaks in the Figure-over a span of $\pm 0.35 \mathrm{~mm}$, in fact. However, due to the column misalignment, mentioned above, the outer left- and right-hand peaks - corresponding to columns 5 and 1 being lit, respectively-were found to be somewhat lower in amplitude than 


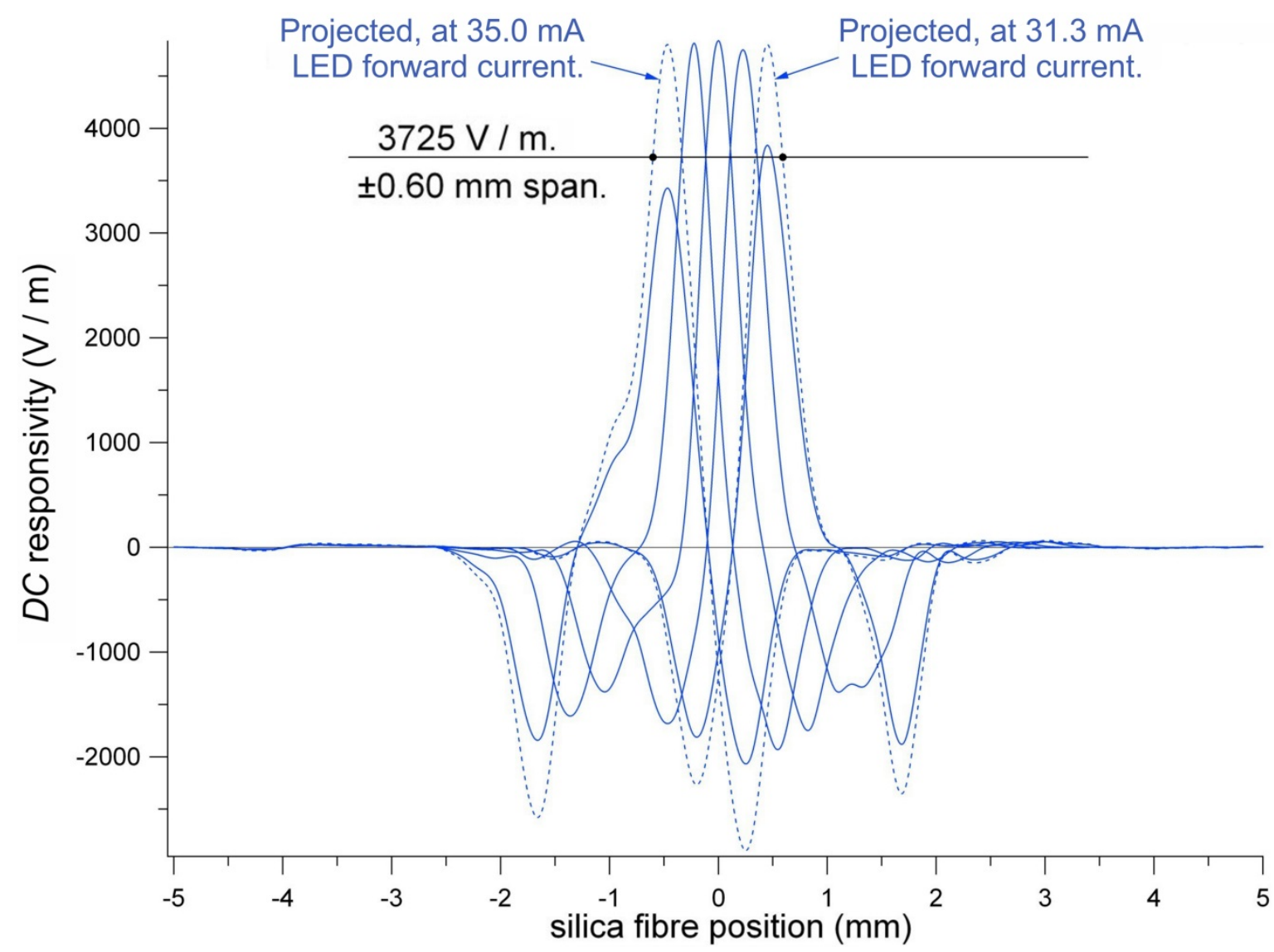

Figure 8. DC responsivity to fibre displacement as a function of mean fibre position. Reading from left-to-right, the five peaks in responsivity (solid traces), clustered around the zero of the abscissa, correspond to LED columns $5,4,3,2$, and 1 being switched on successively, one at a time, using a constant current of $25 \mathrm{~mA}$. Peaks 5 and 1 could have been increased in size to match that of the central three by increasing the bias current to $35.0 \mathrm{~mA}$ (column 5), and $31.3 \mathrm{~mA}$ (column 1) —as shown by the dashed traces—the peak height being closely proportional to the forward current. In that case, a responsivity of at least $3725 \mathrm{~V} / \mathrm{m}$ could have been achieved over a span of $\pm 0.6 \mathrm{~mm}$, as indicated by the section of the horizontal solid line between the two points in the Figure.

the central three. However, the maximum continuous current of OP224 devices is $100 \mathrm{~mA}$, and so the forward currents of columns 5 and 1 could have been increased significantly. Indeed, the measured responsivities were found to be very closely proportional to forward current, and, using this information, the projected responsivities under currents of $35.0 \mathrm{~mA}$ (column 5) and $31.3 \mathrm{~mA}$ (column 1) have been indicated by the dashed traces in Figure 8. A minimum DC responsivity of $3725 \mathrm{~V} / \mathrm{m}$ is now seen to extend over a span of $\pm 0.6 \mathrm{~mm}$, as shown by the two points on the line drawn in the Figure at this level of responsivity.

Figure 9 shows how the peak locations of the traces in Figure 8 varied with the identification number of the active, illuminating, column. In fact, peak responsivity occurred when the vertical axis of the fibre's shadow aligned with the adjacent, common, edge of detector elements PDa and PDb [11]. In other words, Figure 9 shows by how much the fibre's shadow had been stepped across the face of the synthesized split-photodiode detector, by illuminating successive LED columns in the emitter. At an effective mean (optical) distance of $20.0 \mathrm{~mm}$ from the fibre's axis to the sensing-plane of the detector, the measured average step size of $228 \mu \mathrm{m}$ would correspond to a rotational, angular, step size of approximately $0.65^{\circ}$, as anticipated. 


\section{LIGO-P1500195-v2}

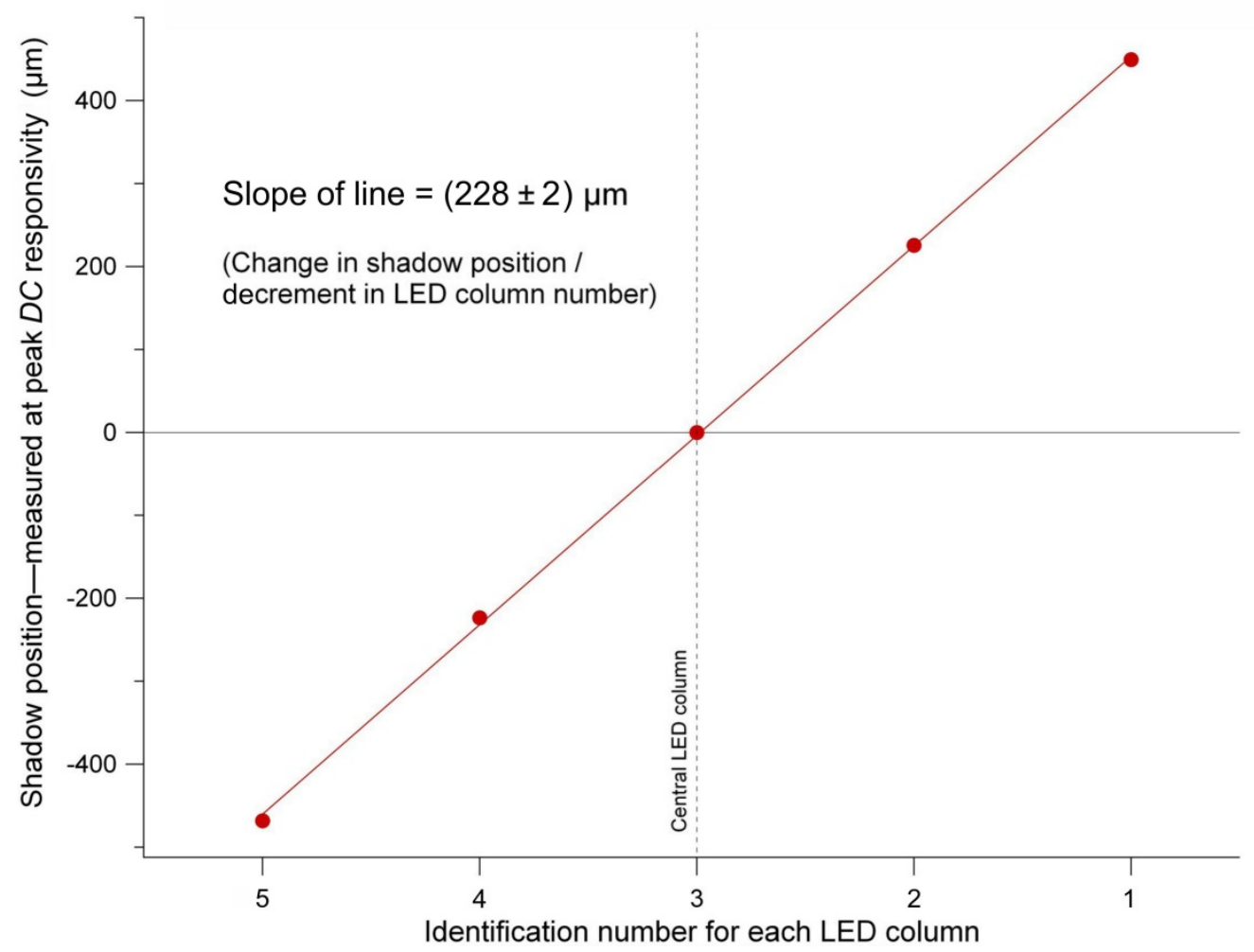

Figure 9. Position of the fibre's shadow as each of the 5 LED columns was used in turn to illuminate the $0.4 \mathrm{~mm}$ diameter silica fibre sample. The measured peak responsivity locations of Figure 8 have been plotted here as a function of the identification number of the (illuminating) LED column. The zero of the shadow position was taken to be the position of peak responsivity for LED column 3.

\section{Gain and noise measurements}

The ratio of the ' $A C(V M)$ gain' to the ' $D C$ gain' of the detector's amplifier was measured to be $904 \pm 4$ at the fundamental $V M$ frequency of $500 \mathrm{~Hz}$ (rather than $\sim 1000$, mid-band) [12,13]. Therefore, a $D C$ responsivity of $3.725 \mathrm{kV} / \mathrm{m}$, as indicated by the horizontal line in Figure 8, corresponded to a mean $A C(V M)$ responsivity to fibre displacement of $904 \times 3725=$ 3.367 MV/m, at $500 \mathrm{~Hz}$. The baseline noise Amplitude Spectral Density for this amplifier had been measured at its $A C(V M)$ output to be $-87 \mathrm{dBV}_{\text {rms }} / \sqrt{\mathrm{Hz}}$ at $500 \mathrm{~Hz}$, in the absence of any photocurrent flowing through either of the photodiode sensing elements, PDa or PDb. However, at the mean (photodiodes equally-shaded) photocurrent level of $12 \mu \mathrm{A}$ encountered with columns 2, 3, or 4 lit, of the 5-column emitter, a shot-noise-limited Amplitude Spectral Density of $(-70.0 \pm 1.0) \mathrm{dBV}_{\mathrm{rms}} / \sqrt{\mathrm{Hz}}$ was measured, at $500 \mathrm{~Hz}$. This is equivalent to an rms (white) noise voltage at $500 \mathrm{~Hz}$ of $(0.318 \pm 0.036) \mathrm{mV} / \sqrt{ } \mathrm{Hz}$, at the amplifier's $\mathrm{VM}$ output.

\section{Conclusions}

At unity signal-to-rms-noise ratio, and using the $A C$ responsivity figure of $3.367 \mathrm{MV} / \mathrm{m}$ found in $\S 7$, the measured noise level of $-70.0 \pm 1.0 \mathrm{dBV}_{\mathrm{rms}} / \sqrt{ } \mathrm{Hz}$ from this same section translates into a $V M$ displacement sensitivity of $(95 \pm 11)$ picometres $/ \sqrt{ } \mathrm{Hz}$, at $500 \mathrm{~Hz}$.

Figure 8 shows the three actual, and two projected, $D C$ (and so, also, $A C$ ) peak responsivities to be $\sim 29 \%$ higher than the level drawn conservatively at $3725 \mathrm{~V} / \mathrm{m}$, upon which the foregoing displacement sensitivity calculation has been based. At these peak values of responsivity, the displacement sensitivity was proportionally higher, of course. If redundancy were sacrificed, the reserve strings of 8 LEDs could be brought into play, yielding an expected increase in displacement sensitivity of a factor $\sqrt{2}$; and, increasing the forward currents of the LEDs in columns 1 and 5, so as to bring their peak responsivities into line with those of columns 2-4, would yield a usefully increased detection span of $\pm 0.6 \mathrm{~mm}$, as mentioned in $\S 6$. Indeed, all of the LED's bias currents could have been increased - to $50 \mathrm{~mA}$, or more, quite comfortably. In 


\section{LIGO-P1500195-v2}

summary, it is clear that the step-wise rotation of the illuminating source functioned as desired, and that it can achieve simultaneously a displacement sensitivity $\sim 100$ picometres (rms)/ $\sqrt{\mathrm{Hz}}$, at $500 \mathrm{~Hz}$, over a measuring span of at least $\pm 0.5 \mathrm{~mm}$, thereby meeting its design targets.

Subsequently, and in the light of tests carried out on actual suspensions, the need to compensate for mechanical creep was relaxed to a working span of just $\pm 0.1 \mathrm{~mm}$, which lay within the scope of the single-LED-column sources of illumination, mentioned in $\S 1$. Four such VM emitters of this type were built and tested successfully, and these, together with their detectors, were measured to have an average VM displacement sensitivity of $(69 \pm 13)$ picometres / $\sqrt{\mathrm{Hz}}$ at $500 \mathrm{~Hz}$, over this span of $\pm 0.1 \mathrm{~mm}[9,11,14]$. However, at the time of writing neither the Violin-Mode emitter/detector system described here, nor its simpler successor, has been adopted for aLIGO, and, indeed, the need for separate VM sensing and damping has not yet been demonstrated. The current baseline solution is to use aLIGO's Arm Length Stabilization system as a VM sensor / damper [15]. In fact, the issue of vacuum compatibility remains unresolved for the VM sensor used in this work, and in its single-emitter-column successor, because the Hamamatsu photodiodes used for the detector elements had been encapsulated, using an unknown epoxy. However, were it to become necessary, the issue of the epoxy for the photodiodes from this, or another, manufacturer probably could be resolved, and the LEDs and other components used are likely to prove vacuum compliant, or have vacuum-compliant alternatives.

\section{Acknowledgements}

We thank the IGR, University of Glasgow, Scotland, UK, particularly Ken Strain and Angus Bell, and Giles Hammond and Rahul Kumar for supplying us with the silica fibre test samples used in this work. Thanks go to Norna Robertson and Calum Torrie of Caltech and the IGR, Alberto Vecchio of the University of Birmingham, and Justin Greenhalgh of the CCLRC (RAL), for their oversight of, and assistance with, this work. We are grateful to the staff of the Physics Department's Electronics Workshop, and the Science Faculty's Mechanical Workshop, at the University of Strathclyde, for their careful construction of most of the component parts used in this work, and we would also like to thank the company Bar Knight Precision Engineers Ltd. of Clydebank, Scotland, for their very good, and timely, machining work. Finally, we are grateful for the support of grant STFC PP/F00110X/1, which sustained this work.

\section{References}

[1] Harry G M (for the LIGO Scientific Collaboration) 2010 Advanced LIGO: the next generation of gravitational wave detectors. Class. Quantum Grav. 27084006 (12pp).

[2] Raab F J et al 2004 Overview of LIGO Instrumentation Proceedings of SPIE 5500 1124 (29 Sept.).

[3] Abbott B P et al 2009 LIGO: The Laser Interferometer Gravitational-Wave Observatory Rep. Prog. Phys. 72076901.

[4] Aston S M et al 2012 Update on quadruple suspension design for Advanced LIGO Class. Quantum Grav. 29235004 (25pp).

[5] Heptonstall A et al 2011 Invited Article: $\mathrm{CO}_{2}$ laser production of fused silica fibers for use in interferometric gravitational wave detector mirror suspensions Rev. Sci. Instrum. 82011301 1-9.

[6] Cumming A V et al 2012 Design and development of the advanced LIGO monolithic fused silica suspension Class. Quantum Grav. 29035003 (18pp).

[7] Carbone, L., et al. 2012 Sensors and actuators for the Advanced LIGO mirror suspensions. Classical and Quantum Gravity 2911115005 (14pp). 


\section{LIGO-P1500195-v2}

[8] Dmitriev A et al 2010 Controlled damping of high-Q violin modes in fused silica suspension fibers. Class. Quantum Grav. 27025009 (8pp).

[9] Lockerbie N A and Tokmakov K V and Strain K A 2014 A source of illumination for low-noise 'Violin-Mode' shadow sensors, intended for use in interferometric gravitational wave detectors Meas. Sci. Technol. 25,12, 12 p., 125111; http://dx.doi.org/10.1088/0957$\underline{0233 / 25 / 12 / 125111 .}$

[10] OP232 and OP224 LEDs: OPTEK Technology (a TT Electronics company), 1645 Wallace Drive, Carrollton, Texas 75006, USA.

[11] Lockerbie N A and Tokmakov K V 2014 A 'Violin-Mode' shadow sensor for interferometric gravitational wave detectors Meas. Sci. Technol. 25, 12, 12 p., 125110; http://dx.doi.org/10.1088/0957-0233/25/12/125110.

[12] Lockerbie N A and Tokmakov K V 2014 A low-noise transimpedance amplifier for the detection of 'Violin-Mode' resonances in advanced LIGO suspensions Rev. Sci. Instrum., 85, 11, 8 p., 114705; http://dx.doi.org/10.1063/1.4900955.

[13] Lockerbie N A and Tokmakov K V 2014 Quasi-static displacement calibration system for a 'Violin-Mode' shadow-sensor in Advanced LIGO suspensions Rev. Sci. Instrum., 85, 10, 12 p., 105003; http://dx.doi.org/10.1063/1.4895640.

[14] Lockerbie N A et al 2011 First results from the 'Violin-Mode' tests on an advanced LIGO suspension, at MIT. Class. Quantum Grav. 28245001 (12pp).

[15] Instrument Science White Paper 2012 LIGO-T1200199-v2, p71 https://dcc.ligo.org. 